\title{
Retrospective analysis of massive epistaxis and pseudoaneurysms in nasopharyngeal carcinoma after radiotherapy
}

\author{
Liang Zeng ${ }^{1}$ \\ ${ }^{1}$ First Affiliated Hospital of Nanchang University
}

April 1, 2021

\begin{abstract}
Objectives This article focuses on massive epistaxis and pseudoaneurysm in patients with NPC after radiotherapy and discusses clinically relevant treatment strategies. Design Retrospective the medical data of NPC patients with massive epistaxis after radiotherapy and review the English literature over the past 10 years. Setting Otorhinolaryngology department in the First Affiliated Hospital of Nanchang University. Participants 21 patients with massive epistaxis after radiotherapy for NPC. Main outcome measures Characteristics and related causes of massive epistaxis or pseudoaneurysms were analyzed in terms of the clinical stage of NPC, course of radiotherapy, and affected artery. An analysis was performed on the methods of endovascular interventional treatment of such pseudoaneurysms. Results 19 cases were accompanied with bone destruction of the skull base; 13 cases were found tumor recurrence; 15 cases were in stage III or IV of NPC; 14 cases were combined with pseudoaneurysms. Analysis with the imaging of pseudoaneurysms, we found that the petrous ICA was the most predilection site. There were 11 out of 14 pseudoaneurysms had sentinel hemorrhage in the initial phase. All 14 pseudoaneurysm patients were underwent endovascular interventional therapy, but one died from hemorrhagic shock during the procedure. There were no rebleeding again among other patients. Conclusions Pseudoaneurysm could cause massive epistaxis with high mortality. The formation of a pseudoaneurysm was closely associated with a high carcinoma stage, re-radiotherapy, and local bone destruction and infection. Most cases had sentinel epistaxis. The imaging material prompted that pseudoaneurysm had a predisposition to the petrous part of the ICA, while the preferred therapy was endovascular embolization treatment. Key poits 1.Patients with massive epistaxis mean a single nasal bleeding volume exceeded $100 \mathrm{ml}$, or cumulative bleeding volume was more than 300 ml. 2.Patients who presented with active oronasal bleeding should be rapidly managed by nasal packing. 3.When there is hemorrhagic shock, patients should undergo endotracheal intubation and blood transfusion before being transferred to the otorhinolaryngology department. 4.CTA and DSA are recommended for patients with massive epistaxis associated with NPC after radiotherapy. 5.Interventional embolization is recommended to patients with pseudoaneurysm.
\end{abstract}

\section{Key poits}

1.Patients with massive epistaxis mean a single nasal bleeding volume exceeded $100 \mathrm{ml}$, or cumulative bleeding volume was more than $300 \mathrm{ml}$.

2.Patients who presented with active oronasal bleeding should be rapidly managed by nasal packing.

3. When there is hemorrhagic shock, patients should undergo endotracheal intubation and blood transfusion before being transferred to the otorhinolaryngology department.

4.CTA and DSA are recommended for patients with massive epistaxis associated with NPC after radiotherapy.

5.Interventional embolization is recommended to patients with pseudoaneurysm.

Abbreviations 
NPC: Nasopharyngeal carcinoma.

CBS: carotid blowout syndrome.

ICA: internal carotid artery.

ECA: external carotid artery.

CCA: common carotid artery.

CTA: computed tomography angiography.

DSA: Digital subtraction angiography.

BOT: balloon occlusion test.

IMRT: Intensity-modulated radiation therapy.

\section{Introduction}

NPC is a common malignant tumor of the nasopharynx, with a high incidence in Southeast Asia and southern China [1]. Radiotherapy, being effective on the biological properties of NPC, is one of the primary treatment modalities. However, there are many radiation-related complications, such as ototoxicity, epistaxis, and pseudoaneurysm. Among them, massive epistaxis after radiotherapy is a serious life-threatening complication. Such patients are usually admitted to the otorhinolaryngology department due to sentinel nasal hemorrhage, which should arouse our attention. We retrospectively analyzed the characteristics and etiological factors of 21 cases of nasopharyngeal hemorrhage in post-irradiated NPC patients and discussed the treatment strategy of endovascular treatment for carotid artery pseudoaneurysm.

\section{Materials and Methods}

We reviewed the medical data of NPC patients with epistaxis after radiotherapy $<$ Blinded for review $>$, from January 2011 to December 2019. Finally, 21 patients were enrolled in our study. Case inclusion criteria were as follows: 1. Patients had a previous history of NPC and underwent one or more courses of radiotherapy without surgery. 2. A single nasal bleeding volume exceeded $100 \mathrm{ml}$, or cumulative bleeding volume was more than $300 \mathrm{ml}$.

In cases of urgent massive bleeding, patients who presented with active oronasal bleeding were rapidly managed by posterior packing. They underwent endotracheal intubation and blood transfusion before being transferred to the otorhinolaryngology department. A few patients underwent tracheotomy because of limited mouth opening. After temporary hemostasis and release of the airway obstruction, patients underwent CTA to identify if there had a carotid pseudoaneurysm. DSA was performed using the transfemoral arterial approach when CTA suggested a pseudoaneurysm. Dynamic images of the internal or external carotid arteries and their branches were assessed for active bleeding with extravasation of the contrast to identify vascular lesions. Guiding catheters were inserted and placed in the lesion segment by DSA, and then the embolic agent was introduced and appropriately deployed. According to the results of the BOT, pseudoaneurysms of the ICA were treated by complete occlusion with a stainless-steel coil or managed with a covered stent. Meanwhile, pseudoaneurysms of the ECA underwent direct embolization of the pseudoaneurysm or occlusion of the pseudoaneurysm-affected artery.

\section{Results}

We summarized the physical and clinical characteristics of the 21 irradiated NPC patients with massive epistaxis. There were 15 males and 6 females with a mean age of 61 years. Persistent epistaxis lasted from a minimum time period of 0.5 hours to a maximum time period of 48 hours. A total of 13 patients underwent one course of radiation therapy, and 8 patients with recurrence underwent re-irradiation. A total of 6 patients of these 21 patients were in stage I, while 15 patients were in stage III or IV of NPC, which was classified according to the seventh edition of the American Joint Committee on Cancer TNM classification. Metastases developed in 5 patients and recurrence occurred in 13 patients. (Table 1). 
CTA findings and the features of pseudoaneurysm of NPC patients:

Routine CTA was performed in all 21 patients, and it showed that 19 patients exhibited extensive skull base destruction. There were 14 patients with pseudoaneurysm; 9 cases were located in the ICA, and 5 cases were located in the ECA. We found that the affected ICAs became obviously narrow according to the CTA after $3 \mathrm{D}$ reconstruction (Figure 1). A total of 11 of 14 pseudoaneurysm patients developed sentinel hemorrhage about three days before the occurrence of massive nasopharyngeal hemorrhage, with single bleeding volume less than $100 \mathrm{ml}$. In terms of the NPC clinical stage, 9 of the 14 pseudoaneurysm patients were in the T3/T4 stage. Meanwhile 9 pseudoaneurysm patients had confirmed local NPC recurrence with pathologic results and 8 patients received re-radiation (Table 2).

Therapy for massive nasopharyngeal hemorrhage and pseudoaneurysms:

Fortunately, all of massive hemorrhage were temporarily controlled after the initial treatment and the patients underwent CTA examination (Figure 2). A total of 7 patients did not present with vascular lesions according to the CTA, and they only received nasal packing. Other 14 pseudoaneurysms were treated by DSA and interventional therapy. Among the 9 ICA pseudoaneurysms, 7 patients underwent occlusion of the ICA with a stainless steel coil (Figure 3) whereas 1 patient failed the BOT to select reconstructive methods with a covered stent to control the hemorrhage. Of the 5 cases of ECA pseudoaneurysms, 2 cases were managed with a steel coil to embolize the parent artery of the pseudoaneurysm. A total of 3 cases directly underwent occlusion of the pseudoaneurysm. All surviving patients did not present with recurrence of epistaxis in the subsequent follow-up at 3 months.

\section{Discussion}

I $n$ recent years, Adaptive IMRT has been reported to reduce the local residual rate of NPC and the occurrence of complications, but it increases the chance of nasopharyngeal hemorrhage [2]. We reviewed some English literatures involving massive epistaxis in NPC after radiotherapy [3-5]. Among these patients, we found that massive epistaxis was one of the main causes of death, and the occurrence of massive epistaxis was associated with infection and necrosis of the nasopharyngeal tissue, radiotherapy, recurrence and re-radiation after recurrence, and pseudoaneurysms. In our study, 19 of the 21 patients presented with osteonecrosis of the skull base and 14 were confirmed to have pseudoaneurysms. To our knowledge, these findings demonstrated that radiation could damage the nasopharynx, skull and wall of the adjacent artery. After continuous radiation, mucosal damage and gland atrophy could occur due to a high radiation dose, which resulted in a decrease in mucus coverage of the nasopharynx. Furthermore, this would gradually trigger the occurrence of mucosal fibrosis, which not only increased the fragility of local blood vessels but also hindered the repair of tissues, eventually causing sustainable ulceration and necrosis [6]. Extensive tissue necrosis and erosion of the skull base bone resulted in the exposure and loss of major blood vessels in the nasopharynx, thereby causing potentially fatal hemorrhage.

In our research, we found that the bleeding volume may exceed $300 \mathrm{ml}$ in an instant. Nevertheless, there were several small amounts of epistaxis before massive hemorrhage. According to our understanding of physiopathologic blood vessel inflammation, small amounts of spontaneous nosebleeds may occur due to rupture of tiny vessels but is stopped by thrombosis, which we called sentinel hemorrhage. Then, we thought the range of rupture of the vascular wall may further expand because of surrounding chronic infection, and it may eventually develop into uncontrollable massive epistaxis. A total of 11 patients of our 14 cases of pseudoaneurysms were found to have sentinel hemorrhage. Therefore, we believed that sentinel hemorrhage was an important characteristic for predicting massive epistaxis after radiotherapy for NPC. Among our 14 pseudoaneurysms, 6 cases received one course of irradiation and 8 cases selected re-radiation due to recurrence of NPC, respectively. This indicated that the occurrence of such pseudoaneurysms was closely related to radiation, which may cause vascular inflammation and lesion, and the damage to the vessel wall caused by radiotherapy was an asymptotic and continuous process.

Compared with DSA, CTA could conveniently identify a pseudoaneurysm in shorter time and less radiation; in the meantime, it can show local stenosis of the ipsilateral carotid artery caused by radiation-induced 
vascular inflammation. Otherwise, CTA can also be used to predict border zone infarction after permanent common carotid artery and/or ICA occlusion by measuring the collateral vessels of the circle of Willis [7]. However, DSA was essential to clarify the sites of pseudoaneurysm, degree of vascular damage, and especially their treatment. The methods and outcomes of endovascular interventions were based on the tumor site, tumor size, and tissue necrosis, as well as the origin of hemorrhage. We favor embolization of the parent artery and performed therapeutic occlusion of the pseudoaneurysm of ECA by coils. And there was no rebleeding. But if the pseudoaneurysm was located in the ICA, the BOT was used to assess the cerebral blood flow, to evaluate the integrity of the circle of Willis. ICA embolization could be performed if the patient passed the BOT; otherwise, a covered stent was required [5]. However, the total occlusion time required for most of the BOT methods, which often last 30-40 minutes, may not be tolerable for CBS patients [8]. Therefore, caution is necessary when patients develop continuous profuse hemorrhage and shock against the standard of BOT. The endovascular management of the CCA had high technical success and allowed immediate hemostasis, which is useful for the initial control of CBS [9]. In the emergency bleeding phase, our experience was that permanent CCA or ICA occlusion with steel coils was an effective treatment with or without passing the BOT. It did produce better clinical results compared with stent-graft placement.

\section{Conclusion}

A pseudoaneurysm occurring in patients with massive epistaxis after radiotherapy for NPC had a high probability with a unique characteristic of sentinel hemorrhage. We considered that the formation of such a pseudoaneurysm was associated with recurrence especially re-radiation, as well as destruction of the nasopharyngeal skull base after radiotherapy. Interventional embolization was the preferred treatment.

\section{Conflicts of interest}

All authors declare no conflicts of interest exist.

\section{References}

[1] Cao S-M, Simons MJ, Qian C-N. The prevalence and prevention of nasopharyngeal carcinoma in China. Chin J Cancer. 2011, 30(2): 114-119.

[2] Dhanachai M, Kraiphibul P, Dangprasert S, et al. Fractionated stereotactic radiotherapy in residual or recurrent nasopharyngeal carcinoma. Acta Oncol, 2007, 46(6): 828-833.

[3] Zhan JB, Zhang S, Wei X, et al. Etiology and management of nasopharyngeal hemorrhage after radiotherapy for nasopharyngeal carcinoma. Cancer Management and Research, 2019, 11: 2171-2178.

[4] Chen HY, Ma XM, Bai YR. Repeated massive epistaxis after re-irradiation in recurrent nasopharyngeal carcinoma. Laryngoscope, 2014, 18(5): 371-376.

[5] Mak C, Cheng KM, Cheung YL, et al. Endovascular treatment of ruptured internal carotid artery pseudoaneurysms after irradiation for nasopharyngeal carcinoma patients. Hong Kong Med J, 2013, 19(3): 229-236.

[6] Kodani N, Yamazaki H, Tsubokura T, et al. Stereotactic body radiation therapy for head and neck tumor: disease control and morbidity outcomes. J Radiat Res, 2011, 52(1): 24-31.

[7] Lee BC, Lin YH, Lee CW, et al. Prediction of borderzone infarction by CTA in patients undergoing carotid embolization for carotid blowout. AJNR Am J Neuroradiol, 2018, 39(7): 1208-1285.

[8] Zussman B, Gonzalez LF, Dumont A, et al. Endovascular management of carotid blowout. World Neurosurg, 2012, 78(1-2): 109-114.

[9] Rodrigues R, Costa J, Anacleto G, et al. Endovascular Treatment of Carotid Blowout Syndrome. Rev Port Cir Cardiotorac Vasc, 2017, 24(3-4): 170.

\section{Hosted file}


\begin\{CJK\}\{UTF8\}\{gbsn\}. \end\{CJK\}\selectlanguage\{english\}pdf available at https://authorea. } com/users/405199/articles/516215-retrospective-analysis-of-massive-epistaxis-andpseudoaneurysms-in-nasopharyngeal-carcinoma-after-radiotherapy

\section{Hosted file}

$\backslash$ begin $\{$ CJK\} $\{$ UTF8\} \{gbsn\}. \end\{CJK\} \selectlanguage\{english\}pdf available at https://authorea. } com/users/405199/articles/516215-retrospective-analysis-of-massive-epistaxis-andpseudoaneurysms-in-nasopharyngeal-carcinoma-after-radiotherapy 